\title{
Are symbols useful and culturally acceptable in health-state valuation studies? An exploratory study in a multi-ethnic Asian population
}

\author{
Hwee-Lin, Wee' \\ Shu-Chuen $\mathrm{Li}^{2}$ \\ Xu-Hao Zhang' \\ Feng Xie ${ }^{3}$ \\ David Feeny ${ }^{4}$ \\ Nan Luo ${ }^{5}$ \\ Yin-Bun Cheung 6 \\ David Machin ${ }^{7}$ \\ Kok-Yong Fong ${ }^{8}$ \\ Julian Thumboo ${ }^{8}$ \\ 'Department of Pharmacy, National \\ University of Singapore (NUS), \\ Singapore; ${ }^{2}$ Discipline of Pharmacy \\ and Experimental Pharmacology, \\ School of Biomedical Sciences, Faculty \\ of Health, University of Newcastle, \\ Newcastle, NSW, Australia; ${ }^{3}$ McMaster \\ University, Hamilton, Ontario, Canada; \\ ${ }^{4}$ Kaiser Permanente Northwest \\ Center for Health Research, \\ Portland, OR, USA; ${ }^{5}$ Department of \\ Community, Occupational and Family \\ Medicine, Yong Loo Lin School of \\ Medicine, NUS, Singapore; ${ }^{6} \mathrm{Clinical}$ \\ Trials and Epidemiology Research \\ Unit, Singapore; ${ }^{7}$ School of Health \\ and Related Research, University of \\ Sheffield, Sheffield, UK; ${ }^{8}$ Department \\ of Rheumatology and Immunology, \\ Singapore General Hospital, Singapore
}

Correspondence: Julian Thumboo Dept of Rheumatology and Immunology, Singapore General Hospital, Outram Road, Singapore 169608

Tel +65632 I 4028

Fax +6562207765

Email julian.thumboo@sgh.com.sg
Background: Symbols have been used in health state valuation studies to help subjects distinguish the severity of various characteristics of a given health state. Symbols used in such studies need to be evaluated for their cross-cultural appropriateness because a given symbol may have different meanings or acceptability in different cultures, which may affect results of such studies.

Objectives: To evaluate if using symbols to differentiate health states of different severity is useful and culturally acceptable in a multi-ethnic, urban Asian population.

Methods: Using in-depth interviews with adult Chinese, Malay, and Indian Singaporeans conducted in English/mother-tongue, subjects were shown a health state with 6 levels (Health Utilities Index 3 vision), each displayed with a symbol, and asked (1a) if symbols were useful in differentiating severity of each level (measured using dichotomous and 0-10 visual analog scale [VAS] scales) or (1b) offensive and (2) to assess 7 alternative sets of symbols.

Results: Of 63 subjects ( $91 \%$ response rate), 18 (29\%) felt symbols were useful in differentiating severity of each level. Reported usefulness of symbols was fair (median VAS score: 3.0, score exceeding 5.0 for $33 \%$ of subjects). One Malay subject felt symbols were offensive.

Conclusions: Use of symbols for health state valuation was culturally acceptable and useful for some subjects.

Keywords: Asian, Southeastern, culture, health status, questionnaires, Singapore

\section{Introduction}

Human communication depends in large part on symbols (Fontana 2003). Individuals constantly come into contact with symbols of various shapes, colors, and sizes. Over time, they begin to develop their own interpretations of these symbols, shaped by culture, values, and experiences (Gesler and Kearns 2002). Some symbols have an almost universal meaning, while others have different meanings in different cultures. For example, circles represent infinity, perfection, and eternity in most cultures (Helfand 2002; London and Recio 2004). In contrast, the chrysanthemum flower represents nobility or autumn in Chinese culture but represents truth (white chrysanthemum) or slighted love (yellow chrysanthemum) in Victorian flower code (Scoble and Field 1998). There also appear to be innate preferences for certain aspects of symbols. For example, Chinese prefer symmetrical symbols such as squares and rectangles to nonsymmetrical symbols, as the former represent balance (Andrews 1993) - an important concept of harmony in the Chinese culture.

Symbols have been used in health-state valuation studies to help subjects distinguish the severity of various characteristics of a given health state (Feeny et al 2002). Symbols used in such studies need to be evaluated for their cross-cultural appropriateness because a given symbol may have different meanings or acceptability in different cultures, which may affect results of such studies. Symbols with strong cultural or 
personal meanings or which are offensive should be avoided, as these may cause subjects to respond based on the symbols themselves, rather than what they were supposed to represent (Uttal et al 1999), thus affecting response rates or data quality. However, to the best of our knowledge, no studies have evaluated the cross-cultural appropriateness of symbols used in health-state valuation studies. As it is unclear if using symbols to differentiate health states of different severity is useful and culturally acceptable among Asians, we studied the subjects' perceptions of these issues in a multi-ethnic, urban Asian population.

\section{Methods}

\section{Subjects}

In this Institutional Review Board-approved study, in-depth interviews in either English or a subject's mother-tongue (ie, Chinese, Malay, or Tamil) using an identical, pre-tested questionnaire were conducted among consenting Chinese, Malay, and Indian Singaporeans with at least 6 years of education by interviewers of the same ethnic group. Subjects with fewer than 6 years of education were not studied as we had previously observed that these subjects had difficulty following instructions in a valuation study protocol. To achieve adequate representation, we recruited 2 male subjects (one speaking English, the other his respective mother tongue) and 2 female subjects (one speaking English, the other her respective mother tongue) from each age band (20-29, 30-39, 40-49, 50-59, >60) from the Singaporean general population, giving a minimum of 20 subjects per ethnic group.

\section{Study design}

This study formed part of a larger study in which subjects participated in an in-depth, one-to-one interview about their views on health states worse than dead, such as chronic or mortal illness, bedridden, or in a coma. Subjects also participated in a health state valuation exercise, and then provided their opinions on the usefulness and cultural acceptability of symbols used in this exercise and 7 alternative symbol sets. Subjects were shown a card listing all 6 levels from the Health Utilities Index 3 (HUI3) vision attribute, where each level was linked with a symbol with increasing severity implied by increasing number of sides in each symbol (Figure 1). Several questions were then asked to determine the usefulness and cultural acceptability of symbols in the health-state valuation process. First, without any prior information, subjects were asked to interpret the purpose of the symbols. After recording subjects' answers, the interviewer explained that the purpose of the symbols was to help differentiate different levels of severity of visual

Able to see well enough to read ordinary newsprint and recognize a friend on the other side of the street, without glasses or contact lenses.

- Able to see well enough to read ordinary newsprint and recognize a friend on the other side of the street, but with glasses.

A Able to read ordinary newsprint with or without glasses but unable to recognize a friend on the other side of the street, even with glasses.

- Able to recognize a friend on the other side of the street with or without glasses but unable to read ordinary newsprint, even with glasses.

+ Unable to read ordinary newsprint and unable to recognize a friend on the other side of the street, even with glasses.

* Unable to see at all.

Figure I Levels for the Health Utilities Index Mark 3 Vision Item.

Note: Increasing severity is indicated by more sides to the corresponding symbol (eg, from 3 sides in a triangle to 6 sides in an asterisk). 
impairment. Second, subjects were asked to evaluate the usefulness of symbols using both dichotomous (yes/no) and 0-10 visual analogue scale (VAS) scales. Third, they were asked if they felt these symbols were culturally acceptable and if any of the symbols were offensive. Finally, they were asked if they preferred an alternative symbol set (Figure 2) using one of the following response options: (a) original set preferred, (b) alternative set preferred, (c) numbers preferred, or (d) no preference. The 7 alternative symbol sets were designed such that severity was reflected by differences in size, shading, and/or color.

\section{Statistical analyses}

Summary statistics were presented as medians with interquartile range (IQR) for continuous variables and percentages with $95 \%$ confidence interval (CI) for categorical variables. Ethnic differences in observations were explored using Chi-squared or Kruskal-Wallis tests. Data were analyzed with STATA (StataCorp 2003).

\section{Results \\ Subjects}

We obtained a response rate of 91\% (63/69 approached subjects). Subjects declined participation because they were busy $(n=2)$ or because the study included a discussion on death $(n=4)$. Among participating subjects, by design, there was an almost equal distribution of Chinese, Malays, and Indians from both genders (Table 1). The median (IQR) age of participants was $43.6(31.9,55.7)$ years and was similar across ethnic groups $(\mathrm{p}=0.76)$. Median $(\mathrm{IQR})$ years of education was $10.0(8.0,13.0)$ and was significantly higher among Chinese subjects $(\mathrm{p}=0.010)$.
(1)
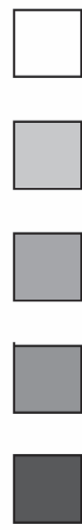

$(n=21)$

(5)
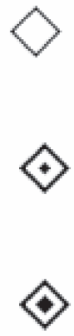

$\square$
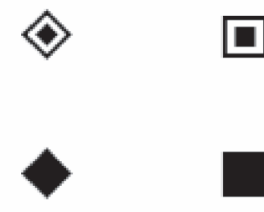

$(n=3,5 \%)$

(2) 0

(6)

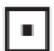

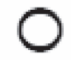

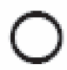

$(n=9,14 \%)$

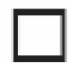

$(n=8,13 \%)$

(7)

a

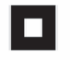

口

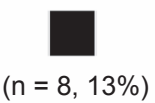

)

$(n=4,6 \%)$
(4)

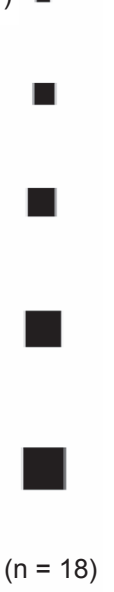

$(n=18)$

Figure 2 Alternative set of symbols.

Notes: I/Increasing severity is indicated by (a) increasing intensity of shadings (Set I), (b) increasing size (Sets 2 to 4) or (c) increasing the areas of black or white within the shape (Sets 5 to 7). 2/Figures in brackets represent number (\%) of subjects indicating a preference for that particular symbol set. 
Table I Characteristics and responses of participants

\begin{tabular}{|c|c|c|c|c|c|}
\hline & \multicolumn{5}{|c|}{$\mathbf{N}(\%)$, unless otherwise specified } \\
\hline & All $(n=63)$ & Chinese $(n=22)$ & Malay $(n=20)$ & Indians $(n=2 I)$ & p value \\
\hline Female & $35(52)$ & $12(55)$ & $10(50)$ & II (52) & 0.96 \\
\hline Median age (IQR) (years) & $43.6(31.9,55.7)$ & $44.6(31.6,56.4)$ & $44.7(30.0,50.6)$ & $40.8(35.1,56.5)$ & 0.76 \\
\hline Years of education & $10.0(8.0,13.0)$ & $13.0(10.0,15.0)$ & $10.0(8.0,12.0)$ & $10.0(8.0,12.0)$ & 0.010 \\
\hline $\begin{array}{l}\text { Interpreted the use of symbols correctly } \\
\text { without prompts }\end{array}$ & $32(5 \mathrm{I})$ & $17(77)$ & $9(45)$ & $6(29)$ & 0.005 \\
\hline (95\% Cl, \%) & $(39-63)$ & $(59-95)$ & $(23-67)$ & $(10-48)$ & \\
\hline Felt symbols were useful & $18(29)$ & $7(32)$ & $7(35)$ & $4(19)$ & 0.48 \\
\hline (95\% Cl, \%) & $(18-40)$ & $(|3-5|)$ & $(14-56)$ & $(2-36)$ & \\
\hline Median (IQR) usefulness of symbols ${ }^{\dagger}$ & $3.0(0,6.0)$ & $3.0(0,6.0)$ & $5.0(1.5,7.8)$ & $3.0(0.5,6.0)$ & 0.37 \\
\hline Felt symbols were offensive & $\mathrm{I}(2)$ & 0 & I (5) & 0 & 0.34 \\
\hline (95\% Cl, \%) & $(0-5)$ & & $(0-15)$ & & \\
\hline $\begin{array}{l}\text { Preferred alternatives for implying } \\
\text { ranking }\end{array}$ & & & & & $<0.001$ \\
\hline No, preferred original symbols & $6(10)$ & 0 & 0 & $6(29)$ & \\
\hline Yes, preferred alternative symbols & $45(7 I)$ & $19(86)$ & $19(95)$ & $7(33)$ & \\
\hline No, preferred numbers & $4(6)$ & 0 & I (5) & $3(14)$ & \\
\hline No, preferred none of the options & $8(13)$ & $3(14)$ & 0 & $5(24)$ & \\
\hline
\end{tabular}

Notes: U Usefulness was rated on a 0 (least useful) to 10 (most useful) visual analogue scale; ₹Subjects may select more than one response. Abbreviations: IQR, interquartile range; $95 \% \mathrm{Cl}$, 95\% confidence interval.

\section{Meaning, usefulness, and acceptability of symbols}

Of 63 subjects, 51\% (95\% CI: 39\%-63\%) understood without prior information that the symbols represented various ranked health levels (Table 1). However, only 29\% (95\% CI: $18 \%-40 \%$ ) felt that the symbols were useful in differentiating the severity of each level of vision. Among all subjects, reported usefulness of symbols was fair (median [IQR] VAS score: 3.0 [0, 6.0]). Of note, 33\% (95\% CI: $21 \%-45 \%)$ of subjects gave a usefulness VAS score exceeding 5.0. All except one Malay subject felt the symbols were culturally acceptable. This lone Malay subject felt the last two symbols (ie, diamond and asterisk sets; Figure 1) were offensive but did not provide further explanations although prompted to do so.

\section{Alternative symbol sets}

When asked to assess alternative sets of symbols, $6(10 \%)$ subjects preferred the original set, $45(71 \%)$ preferred alternative sets, $4(6 \%)$ preferred numbers, and $8(13 \%)$ expressed no preference (Table 1). There were significant ethnic differences $(p<0.001)$ in preference for symbol sets, with fewer Indians preferring alternative sets and no Chinese or Malays preferring the original set. Among these 45 subjects who preferred alternative sets, squares of the same size with increasing shading were preferred by 21 (33\%) subjects, followed by black squares of increasing size (preferred by 18 (29\%) subjects). Seventeen subjects (2 Chinese, 14 Malays, 1 Indian) preferred an alternative set because they felt that comparing different shades and different sizes as an indication of severity was easier and less confusing than comparing the number of sides in a symbol. Another Malay subject felt that different sizes were more useful than different levels of shading to indicate severity. Three subjects (all Indians) preferred numbers because they were easy to understand as they are usually used for ranking purposes. The remaining subjects $(n=28)$ did not elaborate on their choices despite being prompted to do so.

\section{Discussion}

In this study, we evaluated the meaning, usefulness, cultural acceptability, and preference for various sets of symbols for use in health state valuation studies. To the best of our knowledge, this is the first study addressing this issue, and it was encouraging to find that the symbols were generally culturally acceptable in this multi-ethnic Asian population. Interestingly, although symbols were culturally acceptable, less than a third of subjects felt they were useful. A wide 
variety of symbol sets were preferred by individual subjects without any one set being dominant.

The association of visual images (eg, illustrations or pictographs) with verbal and written information has been useful in facilitating recall of information (Waddill and McDaniel 1992; Houts et al 2001) and enhancing physician-patient communication (Moll 1986; Moriyama et al 1994) and was therefore thought to be useful in health state valuation studies where subjects need to integrate several pieces of information. It was thus surprising that not all subjects in our study felt symbols were useful as an adjunct to verbal descriptions of the given health state. One possible explanation is that symbols tend to be abstract compared to illustrations or pictographs, and subjects thus require greater effort to use the information presented by symbols. Hence, subjects with good comprehension of verbal/written information are likely to find symbols less helpful, as may be the case in this study, where subjects had a median of 10 years of education.

As a large majority of subjects preferred the alternative symbol sets, this suggests that usefulness of symbols could be further improved by using different shades or sizes (conceptual framework underlying alternative symbol sets) in designing symbol sets. However, there are potential pitfalls in doing so. Importantly, interval ranking may be implied in some alternative sets (as well as the original set) and bias could potentially be introduced as levels of health in a given health state do not necessarily follow an interval ranking (see Figure 1). For example, using numbers (1, 2, 3, 4, etc), could suggest to subjects that level 2 is twice as severe as level 1 , and level 4 is twice as severe as level 2, etc which are not necessarily the case.

That ethnic differences were observed in preferences for the original versus alternative symbols sets is interesting and supports our hypothesis that symbols carry different meanings in different cultures. While Malay subjects clearly preferred to use shadings/sizes because they are intuitive, reasons for preference for alternative symbols sets among Chinese was less clear. These results provide a basis for further studies to investigate these ethnic differences, which may be important in refining the symbol set(s) used for health valuation and other studies. That none of the Chinese and Malay subjects preferred the original symbol set suggests that this may need to be replaced in the local context.

As the symbol set used may affect the health valuations reported by subjects, the choice of a symbol set needs to be made with care. Given that several symbol sets may be suitable for use in health-state valuation studies in Asia, with some symbol sets preferred by some subgroups (eg, from different ethnic groups), a framework for selecting such symbol sets is needed. This proposed framework should include (but not be limited to) consideration of the following factors, and needs to be validated and refined in further research. First, the symbols should be easily understood by the majority of subjects. Second, interval ranking should not be strongly implied in the symbol set, for reasons discussed above (hence, numbers should clearly not be used). Third, symbols should be culturally acceptable (ie, not offensive). Fourth, the symbols should not carry any special connotations for any particular subgroup. In situations where several ethnic groups are to be studied, as is increasingly common in many countries worldwide, we would suggest identifying the one set of symbols which best meets these criteria across all ethnic groups, so that data are more directly comparable across ethnic groups. Using the results of the current study as an illustration, given that between-subgroup comparability among Chinese, Malays, and Indians is important, then one of the alternative symbol sets could be used since Chinese and Malays preferred them and Indians did not find them offensive. However, should between-subgroup comparability be of secondary interest, then the original symbol set should be used for Indian subjects (since they clearly preferred it) while alternative symbol sets could be used for Chinese and Malay subjects, bearing in mind that this would result in findings being less readily generalizable across studies.

We recognize several limitations of this study. First, the study sample was not drawn at random from the Singapore population, which would be both expensive and difficult for a study of this nature. We therefore specified criteria to ensure equal gender and ethnic representation with a wide age range. Second, as we did not study subjects with fewer than 6 years of education, the generalizability of our findings to these subjects requires confirmation. Nevertheless, it is unclear if subjects with low literacy can participate in health-state valuation studies as previous studies found that successful (ie, nonmissing, logical) responses tend to come from younger and/or better educated subjects (Essink-Bot et al 1993; Dolan and Kind 1996). Third, we examined the usefulness of symbols in distinguishing the levels of severity within vision, one of the 8 attributes of health status of the HUI3 system. These results on the usefulness of symbols do not necessarily generalize to other HUI3 attributes. Fourth, although our investigation provides new evidence, we have not yet tested the usefulness 
of symbols in helping subjects make comparisons among multi-attribute health states.

\section{Conclusions}

In conclusion, we found that use of symbols for health-state valuation was useful and culturally acceptable for some but not all subjects in a multi-ethnic Asian population. Further research is needed to determine reasons for this so as to improve their usefulness/respondent's acceptance in such studies. Based on this study, we have proposed a framework for evaluating and selecting the appropriate symbol set(s) for use in health valuation studies, which takes into account subjects' evaluation of cultural acceptability, ease of comprehension and understanding and perceived measurement properties of these symbols.

\section{Acknowledgments}

We would like to thank Mdm. Halimah Beevi and Ms Syzawani Bte Amrun for their assistance in conducting the interviews. J Thumboo conceived the study, and participated and provided oversight for its design and coordination. HL Wee, YB Cheung, and D Machin participated in the design and coordination of the study and performed the statistical analysis. SC Li, F Xie, XH Zhang, N Luo, D Feeny, and KY Fong participated in the design of the study and its coordination. All authors read and approved the final manuscript.

\section{Disclosure}

This study was funded by a programme grant (03/1/27/18/226) from the Biomedical Research Council of Singapore. It should be noted that David Feeny has a proprietary interest in Health Utilities Incorporated (HUInc.), Dundas, Ontario, Canada. HUInc. distributes copyrighted Health Utilities
Index (HUI) materials and provides methodological advice on the use of HUI.

\section{References}

Andrews T. 1993. Augury and the meaning of landscapes. In: AnimalSpeak: The Spiritual and Magical Powers of Creatures Great and Small. Minnesota: Llewellyn Worldwide, Ch. 5. pp. 57-66.

Dolan P, Kind P. 1996. Inconsistency and health state valuations. Soc Sci Med, 42:609-15.

Essink-Bot ML, Stouthard ME, Bonsel GJ. 1993. Generalizability of valuations on health states collected with the EuroQolc-questionnaire. Health Econ, 2:237-46.

Feeny D, Furlong W, Torrance GW, et al. 2002. Multiattribute and singleattribute utility functions for the health utilities index mark 3 system. Med Care, 40:113-28.

Fontana D. 2003. The Secret Language of Symbols: A visual key to symbols and their meanings. San Francisco: Chronicle Books, pp. 84-110.

Gesler WM, Kearns RA. 2002. Structure and agency. In: Culture/Place/ Health. New York: Routledge, pp. 50-71.

Helfand J. 2002. Reinventing the Wheel. New York: Princeton Architectural Press.

Houts PS, Witmer JT, Egeth HE, et al. 2001. Using pictographs to enhance recall of spoken medical instructions II. Patient Educ Couns, 43:231-42.

London E, Recio B. 2004. In rhythm with the beat of the universe: drumming. In: Sacred rituals: connecting with spirit through labyrinths, sand paintings and other traditional arts. Gloucester, MA: Fair Winds Press, pp. 51-60.

Moll JM. 1986. Doctor-patient communication in rheumatology: studies of visual and verbal perception using educational booklets and other graphic material. Ann Rheum Dis, 45:198-209.

Moriyama M, Harnisch DL, Matsubara S. 1994. The development of graphic symbols for medical symptoms to facilitate communication between health care providers and receivers. Tohoku J Exp Med, 174:387-98.

Scoble G, Field A. 1998. The Meaning of Flowers: Myth, Language and Lore. San Francisco: Chronicle Books LLC, p. 28.

StataCorp. 2003. Stata Statistical Software: Release 8. College Station, TX: StataCorp LP.

Uttal DH, Liu LL, DeLoache JS. 1999. Taking a Hard Look at Concreteness: Do Concrete Objects Help Young Children Learn Symbolic Relations? In: Balter L, Tamis-LeMonda C (eds). Child Psychology: a handbook of contemporary issues. Philadelphia, PA: Psychology Press.

Waddill PJ, McDaniel MA. 1992. Pictorial enhancement of text memory: limitations imposed by picture type and comprehension skill. Mem Cognit, 20:472-82. 